\title{
PGE2 Displays Immunosuppressive Effects During Human Active Tuberculosis
}

\section{Joaquín Pellegrini}

Instituto de Química Biológica de la Facultad de Ciencias Exactas y Naturales (IQUIBICEN). Facultad de Ciencias Exactas y Naturales, Universidad de Buenos Aires. Consejo Nacional de Investigaciones Ci

\section{Candela Martin}

Instituto de Química Biológica de la Facultad de Ciencias Exactas y Naturales (IQUIBICEN). Facultad de Ciencias Exactas y Naturales, Universidad de Buenos Aires. Consejo Nacional de Investigaciones Ci

\section{María Paula Morelli}

Instituto de Química Biológica de la Facultad de Ciencias Exactas y Naturales (IQUIBICEN). Facultad de Ciencias Exactas y Naturales, Universidad de Buenos Aires. Consejo Nacional de Investigaciones Ci

\section{Julieta Aylen Schander}

Laboratorio de Fisiopatología de la Preñez y el Parto, Centro de Estudios Farmacológicos y Botánicos, CONICET-UBA, Buenos Aires

\section{Nancy Liliana Tateosian}

Instituto de Química Biológica de la Facultad de Ciencias Exactas y Naturales (IQUIBICEN). Facultad de Ciencias Exactas y Naturales, Universidad de Buenos Aires. Consejo Nacional de Investigaciones Ci

Nicolás Oscar Amiano

Instituto de Química Biológica de la Facultad de Ciencias Exactas y Naturales (IQUIBICEN). Facultad de Ciencias Exactas y Naturales, Universidad de Buenos Aires. Consejo Nacional de Investigaciones Ci

\section{Agustín Rollandelli}

Instituto de Química Biológica de la Facultad de Ciencias Exactas y Naturales (IQUIBICEN). Facultad de Ciencias Exactas y Naturales, Universidad de Buenos Aires. Consejo Nacional de Investigaciones Ci

\section{Domingo Juan Palmero}

División Tisioneumonología Hospital F.J. Muñiz, Uspallata 2272, (C1282AEN) Buenos Aires

\section{Alberto Levi}

División Tisioneumonología Hospital F.J. Muñiz, Uspallata 2272, (C1282AEN) Buenos Aires

\section{Lorena Ciallella}

División Tisioneumonología Hospital F.J. Muñiz, Uspallata 2272, (C1282AEN) Buenos Aires

\section{María Isabel Colombo}

Instituto de Histología y Embriología de Mendoza, Facultad de Ciencias Médicas, Universidad Nacional de Cuyo-CONICET, (CP 5500) Mendoza

\section{Verónica Edith García ( $\nabla$ vgarcia@qb.fcen.uba.ar)}

Instituto de Química Biológica de la Facultad de Ciencias Exactas y Naturales (IQUIBICEN). Facultad de Ciencias Exactas y Naturales, Universidad de Buenos Aires. Consejo Nacional de Investigaciones Ci 


\section{Research Article}

Keywords: Prostaglandin E2, tuberculosis, patients, host-directed therapy, autophagy

Posted Date: February 15th, 2021

DOI: https://doi.org/10.21203/rs.3.rs-199608/v1

License: (c) (i) This work is licensed under a Creative Commons Attribution 4.0 International License.

Read Full License 


\section{Abstract}

Prostaglandin E2 (PGE2), an active lipid compound derived from arachidonic acid, regulates different stages of the immune response of the host during several pathologies such as chronic infections or cancer. In fact, manipulation of PGE2 levels was proposed as an approach for countering the Type I IFN signature of tuberculosis (TB). However, very limited information regarding the PGE2 pathway in patients with active TB is currently available. In the present work, we demonstrated that PGE2 exerts a potent immunosuppressive action during the immune response of the human host against Mycobacterium tuberculosis (Mtb) infection. Actually, we showed that PGE2 significantly reduced lymphoproliferation, the production of proinflammatory cytokines, and the surface expression of several immunological receptors. On the other hand, PGE2 promoted autophagy in monocytes and neutrophils cultured with Mtb antigens. These results suggest that PGE2 might be attenuating the excessive inflammatory immune response caused by $M t b$, emerging as an attractive therapeutic target. Taken together, our findings contribute to the knowledge of the role of PGE2 in the human host resistance to $M t b$ and highlight the potential of this lipid mediator as a tool to improve anti-TB treatment.

\section{Introduction}

It is estimated that Mycobacterium tuberculosis (Mtb), the etiologic agent of tuberculosis (TB), has killed nearly 1000 million people in the last two centuries ${ }^{1}$. Furthermore, nowadays, TB remains a major global health problem ranking among the top ten causes of death worldwide. Despite the availability of an affordable and effective treatment, TB, alongside with COVID19 in $2020^{2}$, is the leading cause of death from a single infectious agent ${ }^{3}$. Moreover, there are nearly 2 million adult deaths annually from TB in developing countries, accounting for a quarter of the preventable mortality cases ${ }^{3}$. Thus, the main goals of developing new strategies against TB include reduction of treatment length, management of drug resistance's problems, identification of safer treatments and eradication of drug interactions in patients with Human Immunodeficiency Virus (HIV) /TB infection ${ }^{4}$.

Although a vast progress has been made in characterizing the acquired immune response in TB patients, it remains to be elucidated the most appropriate defense mechanisms required to fight $M t b$. The immune response against this pathogen is highly complex, and the outcome of the infection depends at least in part, on the role of several immune mediators with particular temporal dynamics on the host microenvironment. In this regard, in addition to the crucial function of cytokines and chemokines, accumulated evidence in recent years has indicated that different lipid mediators, mainly eicosanoids, are critical in the resolution of mycobacterial infection ${ }^{5,6}$.

Eicosanoids are a family of bioactive lipid mediators derived from arachidonic acid (AA), which is released from membrane phospholipids by phospholipases. There are three main pathways involved in the production of eicosanoids, which typically compete with each other for AA: (i) the cyclooxygenase pathway (COX-1 and COX-2) that produces prostaglandins and thromboxanes, (ii) the pathway of lipoxygenases (5-LO, 12-LO, and 15-LO), that catalyzes the production of leukotrienes and lipoxins, and 
(iii) the cytochrome p40 pathway, which generates hydroxyeicosatetraenoic and epoxieicosatrienoic acids ${ }^{7}$.

The ensuing findings that prostaglandin E (PGE) synthase-deficient mice ${ }^{5}$ and mice lacking the PGE2 receptor EP2 have increased susceptibility to $M t b$ infection ${ }^{8}$ provide strong evidence that the induction of apoptotic death of macrophages by PGE2 is critical for regulating $M t b$ growth in vivo ${ }^{5}$, although the exact mechanisms of PGE2 protection have not been elucidated. In this regard, Chen et al. reported that avirulent $M t b$ strain H37Ra induces PGE2 production, which protects against cell necrosis by preventing the internal mitochondrial membrane damage $\mathrm{e}^{5}$ and promoting a rapid plasma membrane repair ${ }^{9}$. Conversely, high concentrations of PGE2 immunosuppress T cell-mediated immunity against $M t b^{10}$ and contributes to the expansion of regulatory $T$ cells $^{11}$, although the exact role of PGE2 in the development of adaptive immunity during human TB is uncertain.

Administration of both zileuton and PGE2 to IL-1 signaling deficient mice or animals that induced excessive type I Interferon (IFN) showed host-beneficial effects ${ }^{6}$. Inhibition of the enzyme 5lipooxygenase (5-LO) is proposed to shunt eicosanoid production toward increased PGE2 synthesis, which in the context of high-inflammatory environments suppresses detrimental type I IFN production, partially rescues mortality, and reduces bacterial burden ${ }^{6}$. Thus, manipulation of PGE2 and/or 5-LO could counteract these responses in patients with severe TB, opening new avenues to develop host-targeted therapies. Hence, the functional characterization of soluble mediators that participate in the immune response against $M t b$, would be crucial to improve vaccine designs and new immunotherapies that considerably improve control over TB.

Therefore, in this work we investigated the role of PGE2 during the immune response of the human host against Mtb infection. Our main goal was to determine the cellular and physiological bases of PGE2 function to evaluate it as part of a potential host-directed therapy.

\section{Material And Methods}

Subjects.

HIV-uninfected patients with TB were diagnosed at the Servicio de Tisioneumonología Hospital F.J. Muñiz, Buenos Aires, Argentina, based on clinical and radiological data, together with the identification of acid-fast bacilli in sputum. All participating patients had received less than one week of anti-TB regular therapy. Table S1 summarizes the demographic and clinical characteristics of enrolled TB patients. Bacillus Calmette-Guerin (BCG)-vaccinated healthy control individuals (HD) from the community participated in this study. Peripheral blood was collected in heparinized tubes from each participant after obtaining informed consent. The protocols conducted through the present work were approved by the Ethical Committee of Dr. F.J. Muñiz Hospital. All methods were carried out in accordance with relevant guidelines and regulations. 
Exclusion Criteria.

All subjects were 18-60 years old and had no history of illnesses that affect the immune system, such as HIV infection, a recent diagnosis of cancer, treatment with immunosuppressive drugs, hepatic or renal disease, pregnancy, or positive serology for other viral (e.g., hepatitis A, B or C), or bacterial infections (e.g., leprosy, syphilis). Individuals with bleeding disorders or under anticoagulant medication that might be at an increased risk of bleeding during the procedure of obtaining the sample were excluded from the study. Individuals with latent infection were excluded from the present study by using the QuantiFERONTBß GOLD PLUS (Qiagen, 622120 and 622526).

Determination of plasmatic PGE2 levels.

Plasma samples were collected from heparinized peripheral blood from HD and TB patients and centrifuged for $15 \mathrm{~min}$ at $1000 \mathrm{xg}$ at $4^{\circ} \mathrm{C}$. Plasma were then stored at $-70{ }^{\circ} \mathrm{C}$. PGE2 measurement was performed by radioimmunoassay (RIA) as previously described ${ }^{12}$. Radioactivity was measured in a beta scintillation counter. After a logarithmic transformation, the data were expressed as pg of PGE2/ml of plasma. The method has a cross-reactivity of less than $0.1 \%$ and a sensitivity of $5 \mathrm{pg} / \mathrm{tube}$ with a Ka $=1.5$ $\times 10^{10} / \mathrm{mol}$.

Antigen.

In vitro stimulation of cells was performed with a cell lysate from the virulent Mycobacterium tuberculosis strain H37Rv, prepared by mechanic disruption (Mtb-Ag) (BEI Resources, NIAID, NIH: Mycobacterium tuberculosis, Strain H37Rv, whole cell lysate, NR-14822).

Cell preparations and culture conditions.

Peripheral blood mononuclear cells (PBMC) were isolated by centrifugation over Ficoll-Hypaque (GE Healthcare, 17-1440-03). Neutrophils were isolated from heparinized blood by centrifugation on FicollPaque, dextran (Sigma, 31392) sedimentation, and hypotonic lysis ${ }^{13}$. Cells were suspended at $2 \times 10^{6}$ in flat-bottom 24 or 48-well plates with RPMI 1640 (Invitrogen, 22400071) supplemented with L-Glutamine (2mM, Sigma), Penicillin/Streptomycin, and 10\% Fetal Bovine Serum (FBS; Gibco, 10437028) for 16 hours without stimulus to allow monocyte adherence. Cells were then stimulated with $\mathrm{Mtb}(\mathrm{Mtb}-\mathrm{Ag}, \mathrm{BEl}$ Resources, NIH, $10 \mu \mathrm{g} / \mathrm{ml}) \pm$ PGE2 (2 $\mu \mathrm{M}$, Sigma, P0409) \pm IFNa 2a (10 ng/ml, Biosidus) for different time points. In order to determine the effect of different treatments on the autophagic flux, the vacuolar-type HC-ATPase inhibitor Bafilomycin A1 (100 nM; Fermentek, 88899-55-2) was added for the last 2 hours of culture before LC3 determination by flow cytometry.

After isolation, neutrophil preparations were stained with an anti-CD14-PE (Biolegend, 325,608) antibody and analyzed with a FACS Aria II cytometer (BD, San Jose, CA, USA) to guarantee that monocyte contamination was $<0.5 \%$ (Supplementary Figure S3). Viability was corroborated by staining with propidium iodide (PI; BD, 556,547) and by flow cytometry analysis. 
Proliferation assay.

PBMC were stimulated with Mtb-Ag for five days in the presence or absence of PGE2. Cells were pulsed with $\left[{ }^{3} \mathrm{H}\right] \mathrm{TdR}\left(1 \mu \mathrm{Ci} /\right.$ well, Perkin Elmer, MA, EE.UU) and harvested 16 hours later. $\left[{ }^{3} \mathrm{H}\right] \mathrm{TdR}$ incorporation (c.p.m.) was measured in a liquid scintillation counter (Wallac 1214 Rackbeta, Turku, WF, Finland). Proliferation index for each individual was calculated as cpm after Mtb Ag-stimulation/cpm after culturing with medium.

Flow Cytometry.

To determine the expression of immune receptors on lymphocytes, monocytes and neutrophils, cells stimulated with $\mathrm{Mtb}-\mathrm{Ag}(10 \mu \mathrm{g} / \mathrm{ml})$ treated or not with PGE2 $(2 \mu \mathrm{M})$ were blocked in PBS $(137 \mathrm{mM} \mathrm{NaCl}$, $2.7 \mathrm{mM} \mathrm{KCl}^{8} \mathrm{mM} \mathrm{Na}_{2} \mathrm{HPO}_{4}$, and $2 \mathrm{mM} \mathrm{KH}_{2} \mathrm{PO}_{4}$ )-FBS $5 \%$ for $15 \mathrm{~min}$ and then stained for surface expression with fluorophore-marked antibodies against SLAMF1 (BD, 559572), CD31 (BD, 560984$),$ CD80 (Biolegend, 305207), Major Histocompatibility Complex (MHC) -I (Biolegend, HLA-A2, 343306), MHC-II (Biolegend, HLA-DQ, 318106), PD-L1 (eBioscience, MIH1), PD-L2 (BD, MIH18), CD3 (Biolegend, 300306) and CD14 (Biolegend, 367116).

Intracellular staining of endogenous saponin-resistant LC3 was performed as described ${ }^{14}$. Briefly, cells were washed with PBS and then permeabilized with PBS containing $0.05 \%$ saponin. In this protocol, the cells are not fixed, therefore LC3-I is washed out of the cell because, unlike LC3-II, it is not anchored to the autophagosome. Cells were then incubated with mouse anti-human LC3A, B antibody (MBL International, M152-3) for 20 min, rinsed with PBS, incubated with anti-mouse secondary antibody conjugated to fluorescein isothiocyanate (eBioscience,62-6511) for 20 min and rinsed twice with PBS. Afterwards, cells were stained with anti-CD14 or anti-CD3 antibodies (Biolegend, 325608 and 300308) to detect the monocyte and $\mathrm{T}$ lymphocytes populations.

Negative control samples were incubated with an irrelevant isotype-matched monoclonal antibody (Biolegend, 400140). Samples were analyzed on a FACSAria II flow cytometer (BD, San Jose, CA, USA).

ELISA.

Culture supernatants of PBMC stimulated or not with Mtb-Ag in the presence or absence of PGE2 were obtained to evaluate cytokine levels by ELISA. TNFa and IFNy (BioLegend) secretion was measured by ELISA following the manufacturers' instructions.

\section{ROS measurement}

Neutrophils from HD were incubated with 2区,7囚-dichlorofluorescein diacetate (DCFDA, $50 \mu \mathrm{M}$; Invitrogen, D399) for $15 \mathrm{~min}$ at $37^{\circ} \mathrm{C}$. Then, cells were washed and stimulated with or without $\mathrm{Mtb}-\mathrm{Ag}(10 \mu \mathrm{g} / \mathrm{ml}) \pm$ PGE2 $(2 \mu \mathrm{M})$ for $60 \mathrm{~min}$. Finally, DCFDA fluorescence was evaluated to monitor ROS production by flow cytometry. 
Confocal microscopy.

Cells were cultured and stimulated on coverslips for 16 hours. After incubation under different experimental conditions, cells were washed in order to remove non-adherent cells. Adherent cells were then fixed with cold methanol for $20 \mathrm{sec}$, then washed and subsequently permeabilized and blocked with blocking buffer (PBS containing 0.5\% saponin (Santa Cruz Biotechnology, sc-280079A) and 1\% bovine serum albumin (Santa Cruz Biotechnology, sc-2323A) for $15 \mathrm{~min}$. The buffer was afterwards removed and the LC3 primary antibody was added (Cell Signaling Technology, 2775) and incubated for 16 hours at $4^{\circ} \mathrm{C}$. Then, cells were washed with blocking buffer and incubated with the secondary antibody (Alexa Fluor ${ }^{\circledR} 488$ Goat Anti-Rabbit IgG (HCL); Invitrogen, A11008) for 2 hours at room temperature. Finally, nuclei were stained with DAPI. The coverslips were mounted with PBS-glycerol (Sigma-Aldrich, G2025) and fixed cells were imaged using a Zeiss Spectral LSM 510 confocal microscope (Zeiss, Jena, Germany) using objective 63, numerical aperture (NA) 1.42.

Image processing.

All the images were processed using ImageJ software (Wayne Rasband, National Institutes of Health). After the image binarization using a defined threshold, the number of LC3 puncta was quantified using the Particle Analyzer plugin. Brightness and contrast were adjusted in all images belonging to the same individual when needed.

\section{Statistical Analysis.}

Analysis of variance and post hoc multiple comparisons tests were used as indicated in the figure legend. Mann-Whitney $U$ test and Wilcoxon rank sum test were used for the analysis of unpaired and paired samples respectively. $P$ values of $<0.05$ were considered statistically significant.

\section{Results}

We initially determined PGE2 plasma levels in healthy donors (HD) and TB patients by RIA. In line with previous reports ${ }^{6}$, significantly higher concentrations of PGE2 were measured in patients with TB as compared to HD, which might reflect the inflammatory state of the infected individuals (Fig. 1). It is important to mention that a small percentage of patients (15\%) were under treatment with ibuprofen at the recruitment time, given that this is a common medication employed to control fever and other symptoms of TB disease. As expected, due to the inhibitory function of ibuprofen on COX-2 enzyme activity, ibuprofen-treated TB patients displayed lower PGE2 levels as compared to non-treated TB patients (data not shown).

To further investigate the role of PGE2 on the human immune response against Mtb infection, we next studied the effect of this eicosanoid on lymphocyte proliferation. Thus, PBMC from both HD and TB patients were stimulated with an $M t b$ lysate $(M t b-A g)$ in the presence or absence of PGE2 for 5 days. Finally, cell proliferation was analyzed by tritiated thymidine incorporation over the last 16 hours of culture. As shown in Fig. 2A, even at the lowest concentrations PGE2 significantly inhibited cell 
proliferation induced by Mtb-Ag. Furthermore, the suppressive effect of PGE2 on cell proliferation was observed both in HD and TB patients (Fig. 2B).

Subsequently, we decided to evaluate the effect of PGE2 on the expression of surface receptors involved in T lymphocyte activation. Interestingly, we observed that PGE2 treatment significantly decreased SLAMF1 and CD31 expression on CD3 ${ }^{+}$T lymphocytes surface (Fig. 3A, B). Moreover, a marked reduction in the expression of the costimulatory molecule CD80 and MHC class I was also confirmed on CD14 ${ }^{+}$ monocytes treated with this eicosanoid (Fig. 3C, D). Besides, we also detected a slight trend to a decrease in MHC class II expression on HD's cells stimulated with Mtb-Ag in the presence of PGE2 (Fig. 3E). Taken together, these results suggest that PGE2 participates in the regulation of $T$ cell activation in response to mycobacterial antigens.

Additionally, we analyzed the effect of PGE2 on the production of key cytokines that participate in the immune response of the human host against $M t b$. Therefore, we investigated the secretion of IFNY, IL17A, TNFa and IL-1 $\beta$ from PBMC stimulated with $M t b-A g$ in the presence or absence of exogenous PGE2. In line with the results described above, PGE2 induced a significant decrease in IFNY and TNFa production in response to $M$ tb-Ag (Fig. 4A, B). Moreover, PGE2 also diminished IL-1 $\beta$ and IL-17A secretion, although no significant differences were detected (data not shown).

A direct correlation between the number of neutrophils and TB severity disease has been previously described $^{15,16}$. Moreover, a duality between the ability of neutrophils to clear the infection and the contribution of high numbers of these cells to inflammation, disease severity and mortality has been proposed $^{15}$. Thus, we then investigated the effect of PGE2 treatment on the expression of surface receptors on neutrophils from $\mathrm{HD}$ and TB patients. As shown in Fig. 5A, Mtb-Ag stimulation increased the surface expression of SLAMF1 on neutrophils from HD and TB patients as we previously reported ${ }^{16}$. In contrast, antigen stimulation augmented PD-L1 levels only on neutrophils from HD (Fig. 5B). Interestingly, treatment with PGE2 significantly reduced SLAMF1 and PD-L1 expression on neutrophils membrane (Fig. 5B). Moreover, PD-L2 levels were not modulated by Mtb-Ag (Fig. 5C). Of note, we observed that neutrophils from HD downregulated CD11b expression after PGE2 treatment (Supplementary Fig. S1).

Furthermore, we next studied the effect of PGE2 on the intracellular levels of ROS in neutrophils from HD and TB patients. As previously reported, Mtb-Ag stimulation triggered ROS production ${ }^{16}$ (Fig. 5D). Nevertheless, although a slight decrease in ROS production was observed in neutrophils from HD, no significant differences were detected in ROS generation upon PGE2 treatment of human neutrophils (Fig. 5D).

Taken together, the results findings presented so far suggested a suppressive/regulatory role for PGE2 on the innate and adaptive immune responses of the human host against $M t b$. However, different roles for PGE2 during Mtb infection has been described ${ }^{5}$. Accordingly, PGE2 has been proposed as a host-directed treatment to counteract the predominant type I interferon response displayed by severe TB patients ${ }^{6}$ and to protect against cell necrosis 5,9 . 
In this context, autophagy arises as a potential mechanism that may explain the positive role of PGE2 during the early stages of $M t b$ infection. In this regard, it has been shown that cyclic mechanical stretching induces autophagic cell death in tenofibroblasts through the activation of PGE2 production ${ }^{17}$. Moreover, it has been demonstrated that nicotine induces cell stress and autophagy mediated by COX-2 activation and PGE2 production in human colon cancer cells ${ }^{18}$. Furthermore, it was recently described that the expression of COX-2 contributes to increase autophagy and therefore to eliminate Mtb in murine bone marrow-derived macrophages ${ }^{19}$. Nevertheless, this regulation by PGE2 has not been described in primary human cells. Therefore, we next analyzed autophagy modulation by PGE2 in Mtb-Ag stimulatedPBMCs from HD and TB patients.

First, we performed a kinetic study to evaluate the autophagy levels in stimulated-cells from HD and TB patients. As shown in Fig. $6 \mathrm{~A}$ and B, a significant increase in the percentage of CD14+ LC3A, B-II ${ }^{+}$ monocytes and in the relative mean fluorescence intensity ( $\mathrm{MMFI}$ ) were detected in cells treated with PGE2 for 16 hours. However, the augmented levels of autophagy markedly decreased at 24 hours (Fig. 6A, B). The increased LC3A, B-II levels detected at 16 hours were corroborated both in monocytes from HD and TB patients (Fig. 6C). In contrast, no significant differences in LC3A, B-II levels were observed in lymphocytes from both populations of individuals (Fig. 6D). Likewise, confocal microscopy analysis yielded similar results, denoted by a greater number of LC3 puncta per cell in Mtb-Ag stimulatedmonocytes treated with PGE2 as compared to untreated cells (Fig. 6E, F). Interestingly, PGE2 treatment also augmented the percentage of LC3A, B-II+ neutrophils upon Mtb-Ag stimulation (Fig. 6G).

It is important to consider that an increase in LC3-II levels can be associated both with a high synthesis of autophagosomes and with a blockage in the autophagic flow (caused by limited fusion of lysosomes or reduced activity of lysosomal enzymes) that reduces the degradation of this protein. To elucidate the origin of LC3-II accumulation by PGE2 treatment, cells from TB patients were stimulated with Mtb-Ag in the presence or absence of the eicosanoid, and incubated with Bafilomycin A1 (Baf A1) during the last 2 hours of culture. In this way, we were able to corroborate that the addition of Baf A1 causes a significant increase in autophagy levels as compared to monocytes stimulated with Mtb-Ag plus PGE2 (Supplementary Fig. S2). This implies that PGE2 induces the formation of autophagosomes, promoting their maturation and a functional autophagic flow. Therefore, our findings demonstrate that PGE2 causes an autophagy-inducing effect in human monocytes. Interestingly, treatment with PGE2 and Baf A1 produced a significant increase in the percentage of LC3A, B-II lymphocytes as compared to cells only treated with PGE2 (Supplementary Fig. S2), corroborating an autophagy-inducing effect of PGE2 also in this cell type.

Type I IFN have been shown to suppress the production of protective host cytokines after infection with $M t b^{20}$. Furthermore, excessive production of type I IFN is associated with a higher susceptibility to $\mathrm{TB}^{6}$. Interestingly, several reports have described that these cytokines display an autophagy-inducing function in different pathological models such as viral infections and cancer ${ }^{21,22}$. However, so far, the contribution of type I interferons to autophagy modulation had not been studied in the context of human TB. 
Remarkably, IFNa treatment of $M t b-A g$ stimulated-monocytes did not induce a significant increase in the percentage of CD14+ LC3A, B-II cells (Fig. 7A), or in the number of LC3 foci per cell (Fig. 7B, C). However, the combination with PGE2 treatment significantly augmented the levels of autophagy as measured by flow cytometry and immunofluorescence (Fig. 7), highlighting the stimulating role of this lipid mediator in the autophagy process of human monocytes.

Overall, our findings demonstrate a potent immunosuppressive role for PGE2 during innate and adaptive human immune responses against $M t b$ infection, which could contribute to balance an excessive host response and to eliminate mycobacteria by promoting autophagy.

\section{Discussion}

Even though TB is a curable disease with an efficient and economical anti-TB treatment, the current prolonged therapy protocols are difficult to maintain, especially in many regions of the world severely affected by $M t b$ infection ${ }^{23}$. Host-directed therapies might provide an unexploited approach as complementary anti-TB therapies, either by increasing the ability of the host immune system to eliminate mycobacteria or by limiting collateral tissue damage associated with infection. In this regard, it has been suggested that manipulation of PGE2 and/or 5-LO could serve to counteract the IFN type I response in patients with severe TB as a host-directed therapy against $M t b^{6}$.

In this work, we found that TB patients exhibit significantly higher concentrations of plasma PGE2 as compared to HD (Fig. 1). Our findings are in line with other works reporting greater amounts of PGE2 in cerebrospinal fluid from meningeal TB patients as compared to control individuals ${ }^{24}$. Moreover, MayerBarber et al. also observed higher concentrations of PGE2 in plasma from patients with mild TB as compared to HD and even extended this result considering the ratio with LXA-4 production ${ }^{6}$.

Given the multiple functions of PGE2 in various cell types during different stages of the immune response, this eicosanoid is paradoxically a proinflammatory factor with immunosuppressive activity. In fact, we observed that PGE2 treatment inhibited the proliferation of $M t b$-stimulated $T$ cells from HD and TB patients (Fig. 2). In line with our results, it has been shown that treatment with indomethacin, a selective COX-2 inhibitor, increases lymphocyte proliferation in HD and patients with TB or leprosy ${ }^{25}$. Strikingly, Tonby et al. reported that the use of this inhibitor decreases the proliferation and cytokine secretion of mycobacterial antigen-stimulated lymphocytes from TB patients ${ }^{26}$. However, these observations could be linked to a direct inhibitory effect of indomethacin on the activation of the NF-KB pathway ${ }^{26}$.

Optimal T cell activation requires costimulatory signaling provided by the interaction of molecules expressed on the antigen-presenting cell with specific ligands on the Tlymphocyte. Therefore, we also study the effect of PGE2 on the expression of different cell receptors. We observed that 5 days of treatment with PGE2 significantly decreased the expression of SLAMF1 and CD31 on CD3 ${ }^{+}$lymphocytes from HD and TB patients. Accordingly, we previously demonstrated that costimulation through SLAMF1 
increases IFNy expression in cells from TB patients ${ }^{27,28}$. Furthermore, we have also demonstrated that the interaction between CD31 and SAP negatively regulates the pathways that lead to IFNY production during human active pulmonary $\mathrm{TB}^{29}$. Additionally, a reduction in the surface expression of $\mathrm{CD} 80$ and $\mathrm{MHC}$ class I molecules was found on CD14 ${ }^{+}$monocytes treated with PGE2 (Fig. 3). All together, these results demonstrate that PGE2 downregulates the expression of membrane receptors involved in $\mathrm{T}$ cell activation during stimulation with $M t b-A g$. In line with our findings, the addition of PGE2 has been shown to reduce the expression of CD46 on activated T cells ${ }^{30}$. Furthermore, PGE2 also inhibits the expression of CD80, CD86 and MHC class I after LPS stimulation ${ }^{31}$. In contrast, PGE2 might increase dendritic cell maturation in other models ${ }^{32}$, denoting the importance of exposure time and PGE2 concentration, among other variables..

In addition, our studies in human neutrophils revealed a decrease in the surface expression of SLAMF1 and PD-L1 after Mtb-Ag stimulation in the presence of PGE2 (Fig. 5). Previously, we had demonstrated that SLAMF1 promotes ROS generation during Mtb-Ag stimulation of human neutrophils ${ }^{16}$. Nevertheless, here we did not find a significant modulation of ROS production after PGE2 treatment, at least at this time point (60 min). Further investigation is needed to clarify if PGE2 affects other biological functions of neutrophils, such as migration, NET liberation, and phagocytosis, among others.

We also observed that PGE2 treatment significantly reduced IFNy and TNFa secretion from Mtb-Ag stimulated PBMC (Fig. 4). Thus, our data indicate that PGE2 would be affecting the production of critical proinflammatory cytokines required in the immune response against this pathogen. Overall, these results suggest a clear immunosuppressive effect of elevated PGE2 levels during the human immune response against $M t b$.

However, it remains to be understood how PGE2 might collaborate with the host response to contain Mtb, as suggested ${ }^{6}$. Protection against necrosis and the switch towards programmed cell death by apoptosis are key mechanisms in the resolution of an infection. We and other authors have demonstrated that activation of autophagy can result in the elimination of the bacteria in autolysosomes ${ }^{14,33,34}$. Furthermore, autophagy also controls inflammation by regulating signaling pathways of the innate immunity. In fact, autophagy removes endogenous agonists from the inflammasome and modulates the secretion of immune mediators ${ }^{35}$. For these reasons, we next evaluated whether PGE2 could be contributing to an autophagic response during infection with $M$ tb.

Interestingly, by using flow cytometry and confocal microscopy, we showed that treatment with PGE2 increased the levels of autophagy induced in Mtb-Ag stimulated PBMC. Furthermore, the exogenous addition of this eicosanoid triggered a functional autophagy flow both in monocytes and lymphocytes from HD and TB patients (Fig. 6 and Supplementary Figure S2). In different pathological models, including tenofibroblasts and human colon cancer cells, the role of PGE2 in modulating autophagy has been demonstrated ${ }^{17,18,36}$. Furthermore, inhibition of COX-2 has been shown to reverse autophagy induced during lupus nephritis ${ }^{37}$. Besides, kidney autophagy levels were shown to decrease after 
silencing PGES- ${ }^{38}$. Importantly, Xiong et al. reported in murine macrophages that COX-2 suppresses mycobacterial growth by promoting autophagy via the AKT/mTOR pathway ${ }^{19}$. Strikingly, other authors described that PGE2 reverses vitamin D3-induced autophagy in macrophages infected with $\mathrm{Mtb}^{39}$. Nevertheless, further evaluation of autophagy flow is required in those studied cell models.

Remarkably, we detected increased autophagy in lymphocytes stimulated with mycobacterial antigens in the presence of PGE2 plus Baf1 (Supplementary Figure S2). Numerous investigations have shown that autophagy is essential for $\mathrm{CD} 4^{+} \mathrm{T}$ cell survival and homeostasis in peripheral lymphoid organs. Moreover, autophagy is also required for T cell proliferation and cytokine production in response to TCR activation ${ }^{40}$. Besides, autophagy is activated in regulatory $T$ cells and supports lineage stability and survival ${ }^{41}$. Accordingly, it has been previously demonstrated that PGE2 contributes to the expansion of regulatory $T$ cells in response to mycobacterial antigens ${ }^{11}$. Thus, PGE2 activation of autophagy in lymphocytes might directly participate in the preservation of these cells, and could contribute to the generalized immunosuppression observed.

In different models of viral infections, type I IFNs have been found to confer protection against these intracellular pathogens by inducing autophagy ${ }^{42}$. However, most studies support the findings that type I IFNs actually promote infection by $M t b^{43,44}$. Antonelli et al. have shown that IFN receptor type I deficient mice chronically infected with several $M t b$ strains showed reduced bacterial loads compared to WT infected animals ${ }^{45}$. Our analyses by flow cytometry and confocal microscopy revealed that human monocytes stimulated with Mtb-Ag in the presence of IFNa did not modify autophagy levels (Fig. 7). However, exogenous addition of PGE2, even in the presence of elevated type I IFN concentrations significantly induced autophagy in those cells (Fig. 7). This finding reinforces the role of PGE2 as an inducer of monocyte autophagy, especially considering that type I IFNs promote an IFNY-refractory macrophage phenotype that inhibits autophagy and stimulates mycobacteria growth ${ }^{46}$.

Rangel Moreno et al. have described a differential participation of this lipid mediator during the early and late phases of experimental pulmonary $\mathrm{TB}^{10}$. Thus, the inhibitory or stimulating effect of PGE2 might vary during the acute and chronic stages of TB infection. Moreover, Sorgi et al. demonstrated that COX-2 inhibition significantly reduced PGE2 levels, enhanced IFN-y production and NO release, and increased macrophage phagocytosis of $M t b$, reinforcing the notion that optimal PGE2 levels are required for effective modulation of the immune response against $M t b$ infection ${ }^{47}$. Thus, during the design of new personalized treatments focused on the regulation of the levels of host eicosanoids, these various consequences will have to be considered.

In summary, our findings suggest that PGE2 displays a main suppressive role during innate and adaptive immunity against $M t b$, participating in the homeostasis of the immune response and protecting the host from excessive inflammation. Moreover, we hypothesized that PGE2-induced autophagy could be involved in immunity suppression and bacterial clearance. 


\section{Declarations}

\section{Acknowledgements}

We are thankful to Licenciado Guillermo Piazza for his constant support and technical assistance.

J.M.P. and V.E.G. designed the study. J.M.P. and C.M. performed the experiments. J.M.P, C.M. and M.P.M. were responsible for processing samples and performing data management and analysis. J.A.S., N.L.T., A.R. and N.O.A. contributed with standard laboratory work and discussion. A.L., L.C. and D.J.P. were in charge of patients' diagnosis and obtaining the blood samples. M.I.C. provided expert advice. J.M.P. and V.E.G. wrote the manuscript. All authors contributed to data gathering and interpretation, and revision of the report.

This work was supported by the Agencia Nacional de Promoción Científica y Tecnológica [PICT-20150611]; Agencia Nacional de Promoción Científica y Tecnológica [PICT-2017-1451]; Secretaria de Ciencia y Técnica, Universidad de Buenos Aires [20020170100127BA].

\section{References}

1. Daniel, T. M. The history of tuberculosis. Respir. Med. 100, 1862-1870 (2006).

2. World Health Organization. WHO coronavirus disease (COVID-19) dashboard.

3. Hughes, D. \& Karlén, A. Discovery and preclinical development of new antibiotics. Ups. J. Med. Sci. 119, 162-169 (2014).

4. WHO | Global tuberculosis report 2019. https://www.who.int/tb/publications/global_report/en/.

5. Chen, M. et al. Lipid mediators in innate immunity against tuberculosis: opposing roles of PGE2 and LXA4 in the induction of macrophage death. J. Exp. Med. 205, 2791-2801 (2008).

6. Mayer-Barber, K. D. et al. Host-directed therapy of tuberculosis based on interleukin-1 and type I interferon crosstalk. Nature 511, 99-103 (2014).

7. Dietzold, J., Gopalakrishnan, A. \& Salgame, P. Duality of lipid mediators in host response against Mycobacterium tuberculosis: good cop, bad cop. F1000prime Rep. 7, 29 (2015).

8. Kaul, V. et al. An Important Role of Prostanoid Receptor EP2 in Host Resistance to Mycobacterium tuberculosis Infection in Mice. J. Infect. Dis. 206, 1816-1825 (2012).

9. Divangahi, M. et al. Mycobacterium tuberculosis evades macrophage defenses by inhibiting plasma membrane repair. Nat. Immunol. 10, 899-906 (2009).

10. Rangel Moreno, J. et al. The role of prostaglandin E2 in the immunopathogenesis of experimental pulmonary tuberculosis. Immunology 106, 257-266 (2002).

11. Garg, A. et al. Mannose-capped lipoarabinomannan- and Prostaglandin E2-dependent expansion of regulatory T cells in human Mycobacterium tuberculosis infection. Eur. J. Immunol. 38, 459-469 (2008). 
12. Ribeiro, M. L. et al. Epidermal growth factor prevents prepartum luteolysis in the rat. Proc. Natl. Acad. Sci. 102, 8048-8053 (2005).

13. Gabelloni, M. L. et al. NADPH oxidase derived reactive oxygen species are involved in human neutrophil IL-1 $\beta$ secretion but not in inflammasome activation. Eur. J. Immunol. 43, 3324-3335 (2013).

14. Tateosian, N. L. et al. IL17A augments autophagy in Mycobacterium tuberculosis-infected monocytes from patients with active tuberculosis in association with the severity of the disease. Autophagy 13, 1191-1204 (2017).

15. Kroon, E. E. et al. Neutrophils: Innate Effectors of TB Resistance? Front. Immunol. 9, 2637 (2018).

16. Pellegrini, J. M. et al. Neutrophil autophagy during human active tuberculosis is modulated by SLAMF1. Autophagy 1-10 (2020) doi:10.1080/15548627.2020.1825273.

17. Chen, H., Chen, L., Cheng, B. \& Jiang, C. Cyclic mechanical stretching induces autophagic cell death in tenofibroblasts through activation of prostaglandin E2 production. Cell. Physiol. Biochem. Int. J. Exp. Cell. Physiol. Biochem. Pharmacol. 36, 24-33 (2015).

18. Pelissier-Rota, M. A., Pelosi, L., Meresse, P. \& Jacquier-Sarlin, M. R. Nicotine-induced cellular stresses and autophagy in human cancer colon cells: A supportive effect on cell homeostasis via upregulation of Cox-2 and PGE2 production. Int. J. Biochem. Cell Biol. 65, 239-256 (2015).

19. Xiong, W. et al. Novel Function of Cyclooxygenase-2: Suppressing Mycobacteria by Promoting Autophagy via the Protein Kinase B/Mammalian Target of Rapamycin Pathway. J. Infect. Dis. 217, 1267-1279 (2018).

20. McNab, F., Mayer-Barber, K., Sher, A., Wack, A. \& O'Garra, A. Type I interferons in infectious disease. Nat. Rev. Immunol. 15, 87-103 (2015).

21. Schmeisser, H., Bekisz, J. \& Zoon, K. C. New function of type I IFN: induction of autophagy. J. Interferon Cytokine Res. Off. J. Int. Soc. Interferon Cytokine Res. 34, 71-78 (2014).

22. Zhao, J. et al. Interferon-alpha-2b induces autophagy in hepatocellular carcinoma cells through Beclin1 pathway. Cancer Biol. Med. 11, 64-68 (2014).

23. The Lancet Respiratory Medicine Commission: 2019 update: epidemiology, pathogenesis, transmission, diagnosis, and management of multidrug-resistant and incurable tuberculosis - The Lancet Respiratory Medicine. https://www.thelancet.com/journals/lanres/article/PIIS22132600(19)30263-2/fulltext.

24. Abdulaziz, A. T. A., Li, J. \& Zhou, D. The prevalence, characteristics and outcome of seizure in tuberculous meningitis. Acta Epileptol. 2, 1 (2020).

25. Bahr, G. M., Rook, G. A. \& Stanford, J. L. Prostaglandin-dependent regulation of the in vitro proliferative response to mycobacterial antigens of peripheral blood lymphocytes from normal donors and from patients with tuberculosis or leprosy. Clin. Exp. Immunol. 45, 646-653 (1981).

26. Tonby, K. et al. The COX- inhibitor indomethacin reduces Th1 effector and T regulatory cells in vitro in Mycobacterium tuberculosis infection. BMC Infect. Dis. 16, 599 (2016). 
27. Pasquinelli, V. et al. IFN-y Production during Active Tuberculosis Is Regulated by Mechanisms That Involve IL-17, SLAM, and CREB. J. Infect. Dis. 199, 661-665 (2009).

28. Quiroga, M. F. et al. Activation of signaling lymphocytic activation molecule triggers a signaling cascade that enhances Th1 responses in human intracellular infection. J. Immunol. Baltim. Md 1950 173, 4120-4129 (2004).

29. Quiroga, M. F. et al. Cross-Talk between CD31 and the Signaling Lymphocytic Activation MoleculeAssociated Protein during Interferon-y Production against Mycobacterium tuberculosis. J. Infect. Dis. 196, 1369-1378 (2007).

30. Kickler, K. et al. Prostaglandin E2 affects T cell responses through modulation of CD46 expression. J. Immunol. Baltim. Md 1950 188, 5303-5310 (2012).

31. Jung, I. D. et al. COX-2 and PGE2 signaling is essential for the regulation of IDO expression by curcumin in murine bone marrow-derived dendritic cells. Int. Immunopharmacol. 10, 760-768 (2010).

32. Kalinski, P. Regulation of Immune Responses by Prostaglandin E2. J. Immunol. Baltim. Md 1950 188, 21-28 (2012).

33. Castillo, E. F. et al. Autophagy protects against active tuberculosis by suppressing bacterial burden and inflammation. Proc. Natl. Acad. Sci. 109, E3168-E3176 (2012).

34. Gutierrez, M. G. et al. Autophagy Is a Defense Mechanism Inhibiting BCG and Mycobacterium tuberculosis Survival in Infected Macrophages. Cell 119, 753-766 (2004).

35. Deretic, V. \& Levine, B. Autophagy balances inflammation in innate immunity. Autophagy 14, $243-$ 251 (2018).

36. Martínez-Colón, G. J. \& Moore, B. B. Prostaglandin E2 as a Regulator of Immunity to Pathogens. Pharmacol. Ther. 185, 135-146 (2018).

37. Jin, J. et al. Activation of Cyclooxygenase-2 by ATF4 During Endoplasmic Reticulum Stress Regulates Kidney Podocyte Autophagy Induced by Lupus Nephritis. Cell. Physiol. Biochem. Int. J. Exp. Cell. Physiol. Biochem. Pharmacol. 48, 753-764 (2018).

38. Li, T. et al. Aggravation of acute kidney injury by mPGES-2 down regulation is associated with autophagy inhibition and enhanced apoptosis. Sci. Rep. 7, 10247 (2017).

39. Wan, M. et al. Prostaglandin E2 suppresses hCAP18/LL-37 expression in human macrophages via EP2/EP4: implications for treatment of Mycobacterium tuberculosis infection. FASEB J. Off. Publ. Fed. Am. Soc. Exp. Biol. 32, 2827-2840 (2018).

40. Jacquin, E. \& Apetoh, L. Cell-Intrinsic Roles for Autophagy in Modulating CD4 T Cell Functions. Front. Immunol. 9, (2018).

41. Zhang, J. et al. Autophagy in regulatory T cells: A double-edged sword in disease settings. Mol. Immunol. 109, 43-50 (2019).

42. Tian, Y., Wang, M.-L. \& Zhao, J. Crosstalk between Autophagy and Type I Interferon Responses in Innate Antiviral Immunity. Viruses 11, (2019). 
43. Flynn, J. L. \& Chan, J. What's good for the host is good for the bug. Trends Microbiol. 13, 98-102 (2005).

44. McNab, F. W. et al. TPL-2-ERK1/2 Signaling Promotes Host Resistance against Intracellular Bacterial Infection by Negative Regulation of Type I IFN Production. J. Immunol. 191, 1732-1743 (2013).

45. Antonelli, L. R. V. et al. Intranasal Poly-IC treatment exacerbates tuberculosis in mice through the pulmonary recruitment of a pathogen-permissive monocyte/macrophage population. J. Clin. Invest. 120, 1674-1682 (2010).

46. Lienard, J., Movert, E., Valfridsson, C., Sturegård, E. \& Carlsson, F. ESX-1 exploits type I IFN-signalling to promote a regulatory macrophage phenotype refractory to IFNY-mediated autophagy and growth restriction of intracellular mycobacteria. Cell. Microbiol. 18, 1471-1485 (2016).

47. Sorgi, C. A. et al. Eicosanoid pathway on host resistance and inflammation during Mycobacterium tuberculosis infection is comprised by LTB4 reduction but not PGE2 increment. Biochim. Biophys. Acta BBA - Mol. Basis Dis. 1866, 165574 (2020).

\section{Figures}




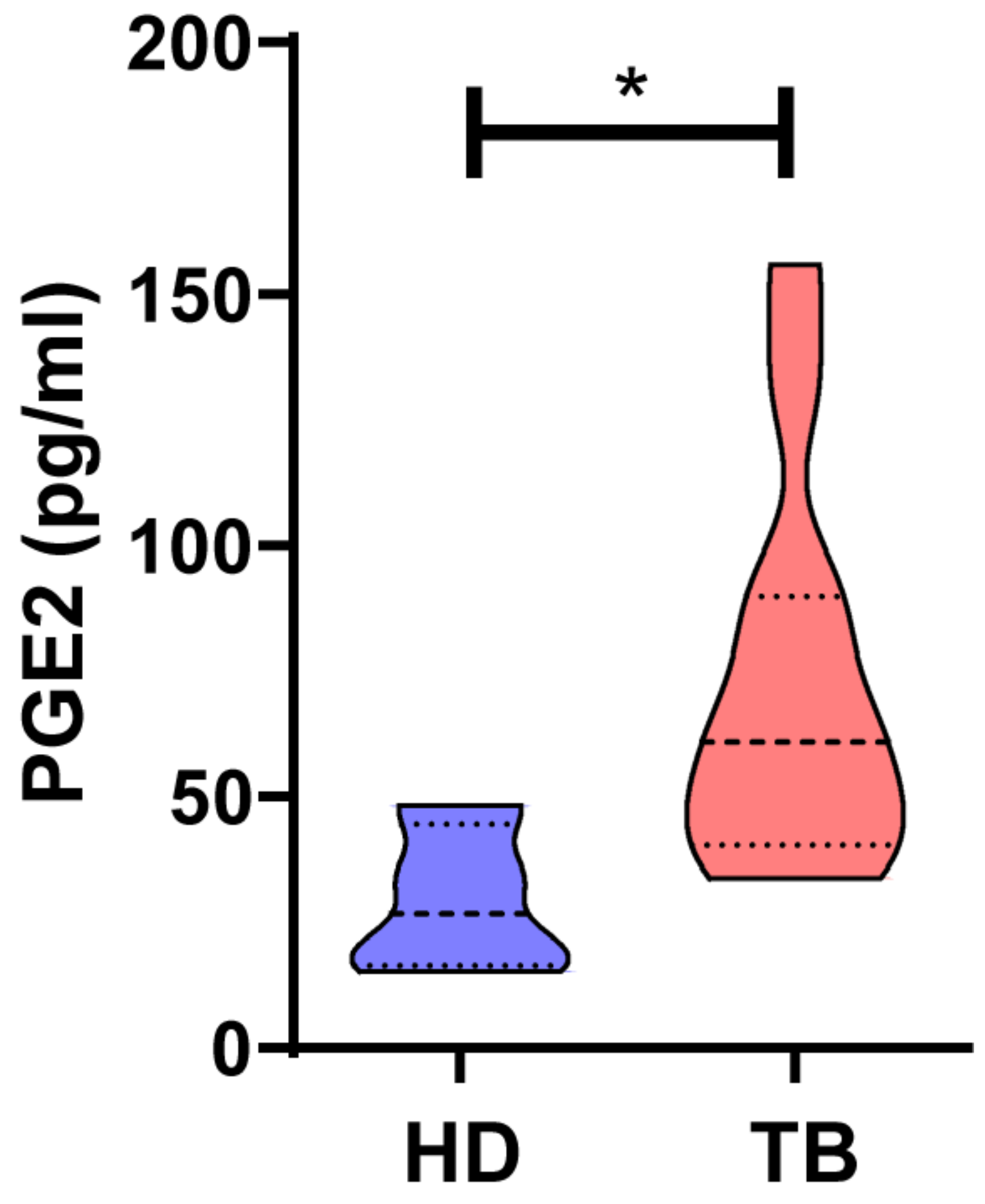

Figure 1

PGE2 plasma levels from Healthy Donors and tuberculosis patients. Heparinized peripheral blood from HD $(n=5)$ and TB patients $(n=10)$ was centrifuged for 15 min at $1000 \mathrm{~g}$ and the levels of PGE2 in plasma were analyzed by RIA. Violin plots show the median values of PGE2 plasma concentration $(\mathrm{pg} / \mathrm{ml}) \pm$ interquartile range. $P$ values were calculated using the Mann Whitney non-parametric test for unpaired samples. ${ }^{*} p<0.05$. 


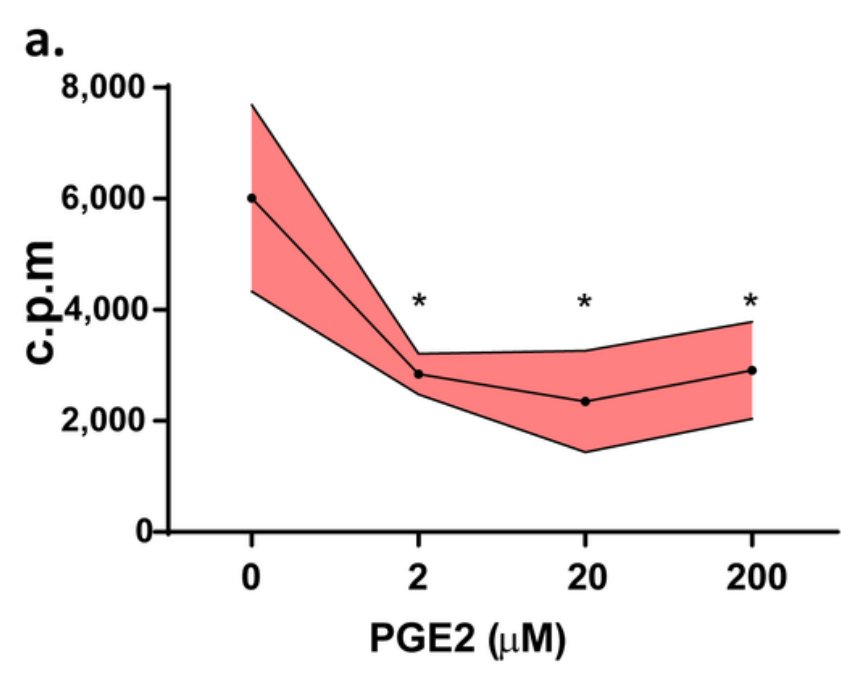

b.

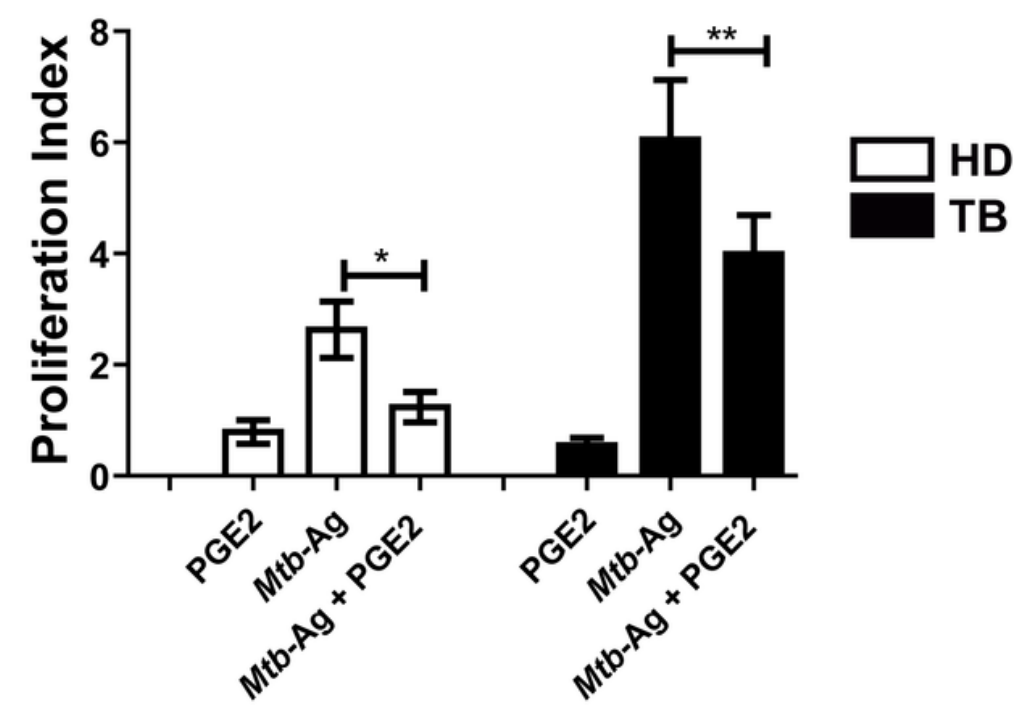

Figure 2

PGE2 inhibits lymphocyte proliferation. PBMC from TB patients and/or HD were stimulated with Mtb-Ag $(10 \mu \mathrm{g} / \mathrm{ml})$ in the presence or absence of for 5 days. Afterwards, cellular proliferation was analyzed by the incorporation of titrated thymidine during the last 16 hours of culture. A) Dose-response curve of PGE2 (0$200 \mu \mathrm{M})$ on proliferation of PBMC from HD $(n=7)$. (B) Effect of PGE2 $(2 \mu M)$ on proliferation from HD $(n=7)$ and TB patients $(n=6)$. Bars show the values of proliferation index (c.p.m. in each condition/c.p.m. media) \pm SEM. Statistical differences were calculated using one-way ANOVA and post hoc Dunnett's multiple comparison test. ${ }^{\star} p<0.05,{ }^{*} p<0,01$. 

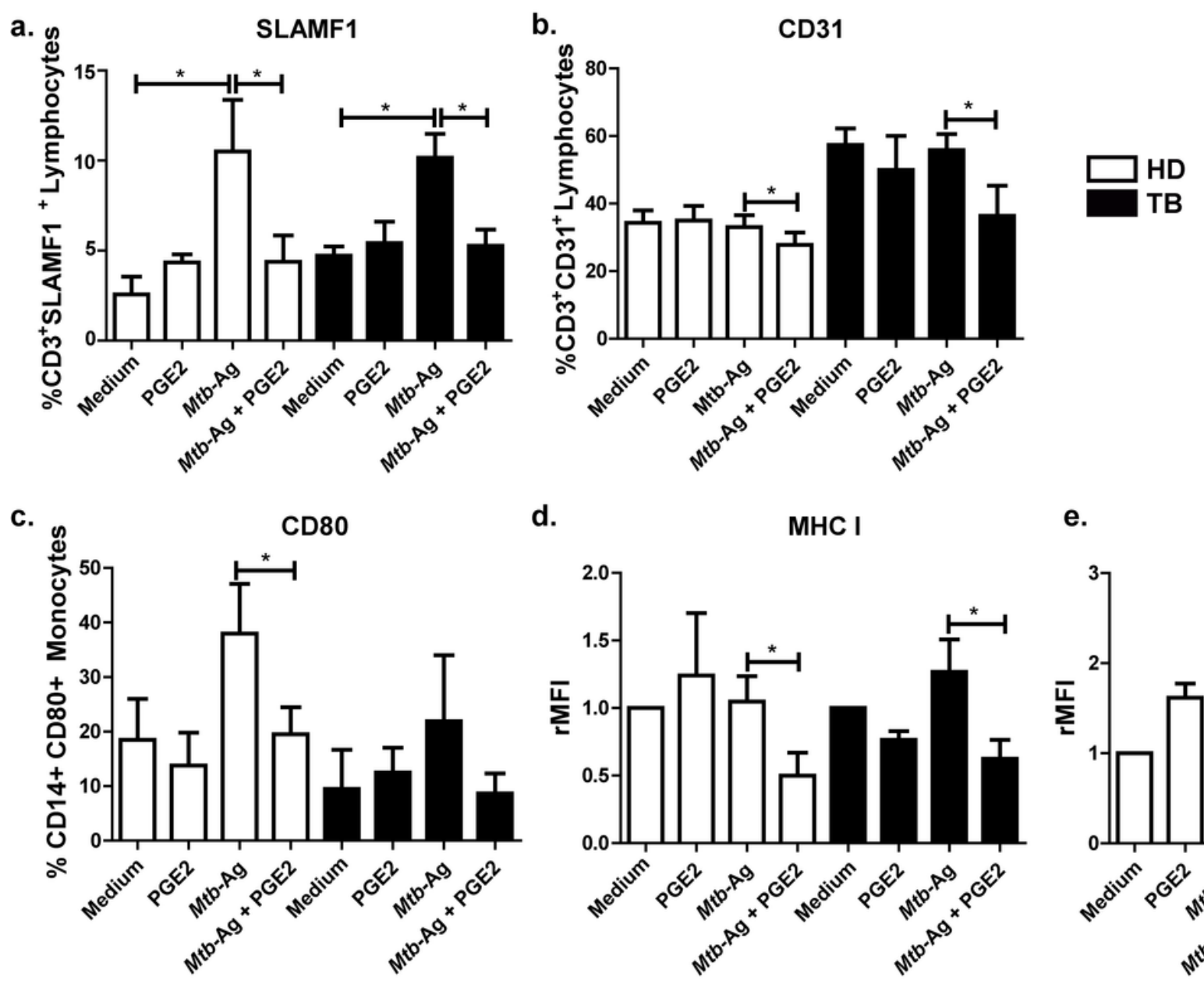

d.

MHC I

e. $\quad$ MHC II
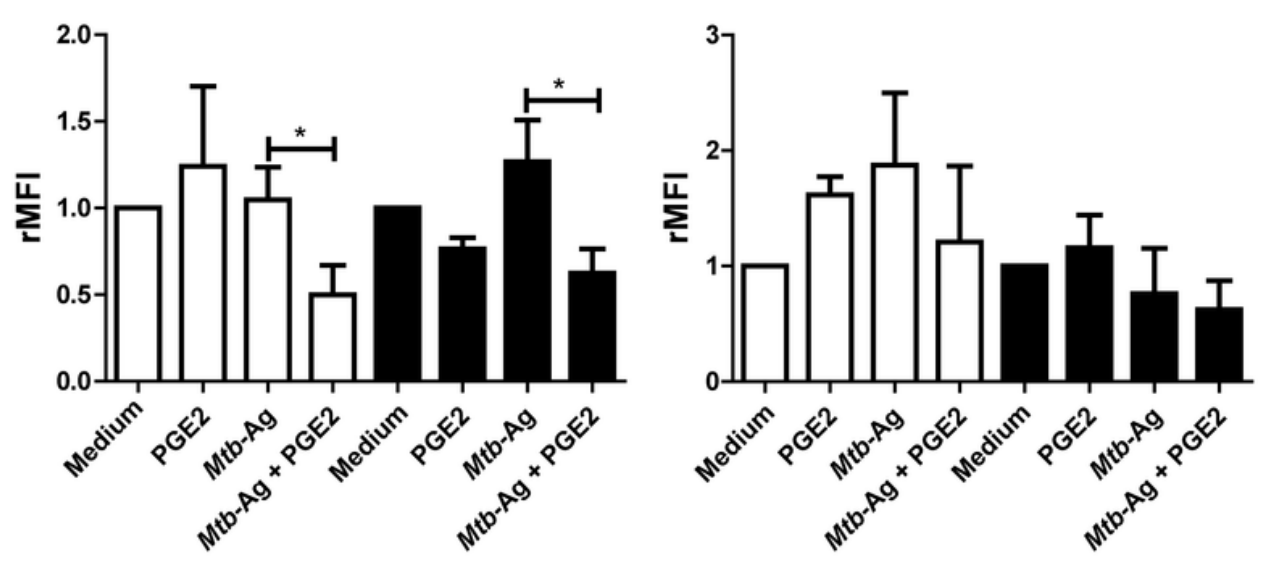

Figure 3

PGE2 regulates the expression of surface receptors on PBMC from HD and TB patients. PBMC from HD $(n=7)$ and TB patients $(n=11)$ were stimulated with Mtb-Ag $(10 \mu \mathrm{g} / \mathrm{ml})$ in the presence or absence of PGE2 $(2 \mu \mathrm{M})$ for 5 days. Afterwards, the surface expression of (A) SLAMF1 and (B) CD31 on CD3+ T cells and (C) CD80, (D) MHC-I and (E) MHC-II on CD14+ monocytes were determined by flow cytometry. Each bar represents the mean percentage of $(A, B, C) C D 3+$ or $(D, E) C D 14+$ Receptor+ cells \pm SEM or relative Mean Fluorescence Intensity (rMFI) \pm SEM. Statistical differences were calculated using one-way ANOVA and post hoc Dunnett's multiple comparison test. ${ }^{*} p<0.05$. 

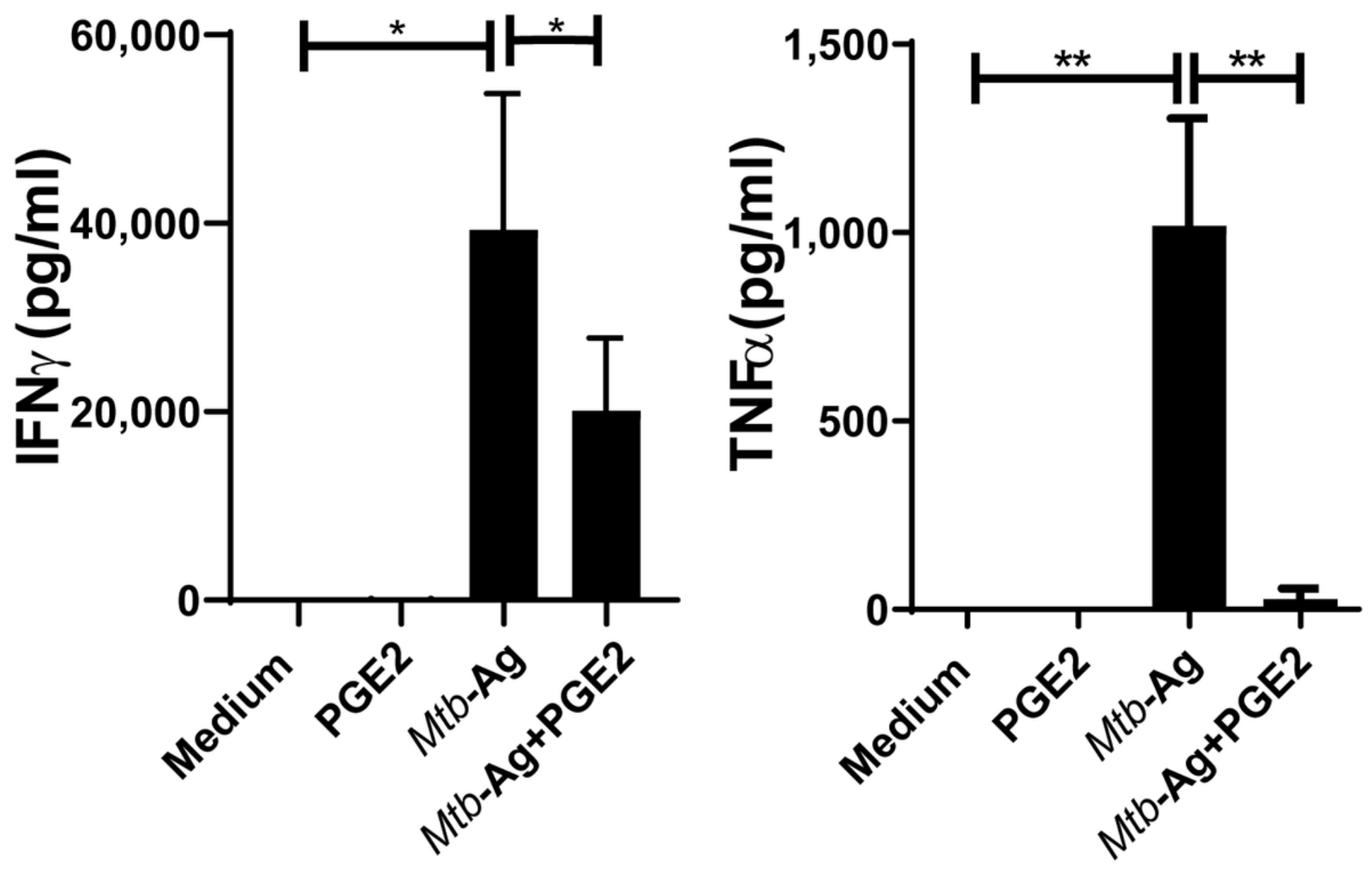

Figure 4

PGE2 modulates cytokine production by PBMC from TB patients. PBMC from TB patients $(n=4)$ were stimulated with Mtb-Ag $(10 \mu \mathrm{g} / \mathrm{ml})$ in the presence or absence of PGE2 $(2 \mu \mathrm{M})$ for (A) 5 days or (B) 24 hours. Afterwards, the production of (A) IFN $y$ and (B) TNF a were determined by ELISA. Each bar represents the mean of cytokine production $(\mathrm{pg} / \mathrm{ml}) \pm \mathrm{SEM}$. Statistical differences were calculated using one-way ANOVA and post hoc Dunnett's multiple comparison test. * $p<0.05,{ }^{\star *} p<0.01$. 
a.

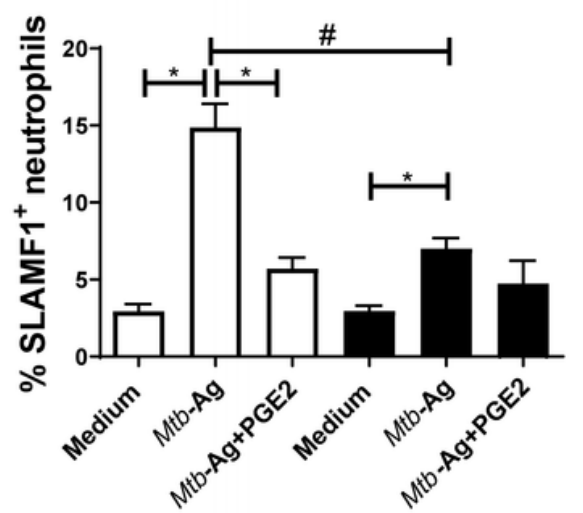

d.

b.

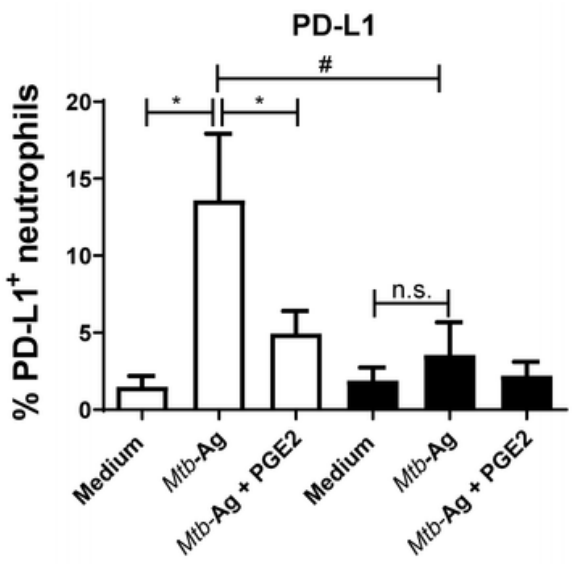

c.

PD-L2

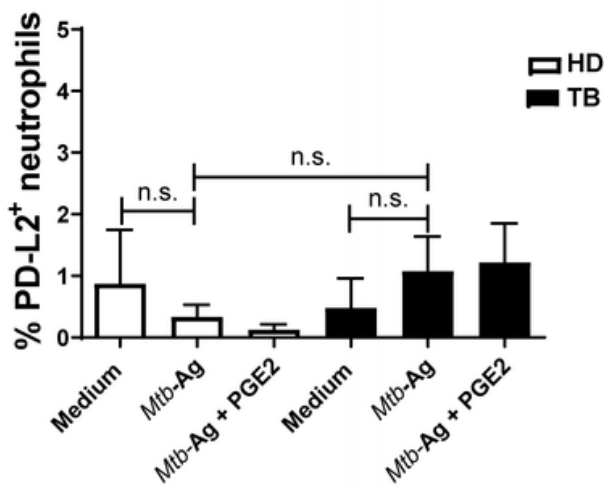

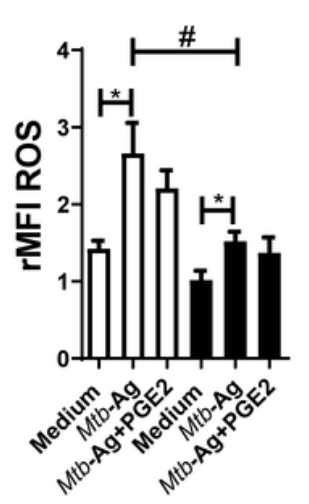

ROS

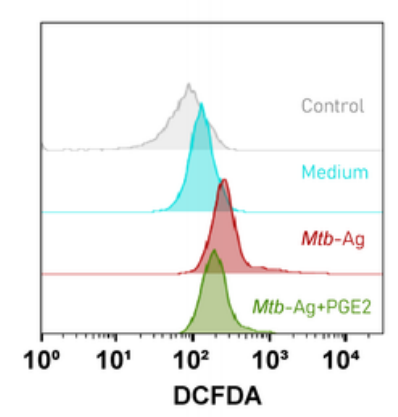

Figure 5

PGE2 regulates the expression of surface receptors on human neutrophils. Human purified neutrophils from HD $(n=7)$ and TB patients $(n=6)$ were stimulated with Mtb-Ag $(10 \mu \mathrm{g} / \mathrm{ml})$ in the presence or absence of PGE2 $(2 \mu \mathrm{M})$ for 2 hours. Then, (A) SLAMF1, (B) PD-L1 and (C) PD-L2 expression were evaluated by flow cytometry. Bars represent the mean values of the percentage of Receptor+ neutrophils \pm SEM. Statistical differences were calculated using one-way ANOVA and post hoc Holm-Sidak's multiple comparison test. ${ }^{*}<<0.05, \# p<0.05$; Mann-Whitney nonparametric test for unpaired samples. (D) Neutrophils from HD $(n=5)$ and TB patients $(n=5)$ were incubated with 2区,7区-dichlorofluorescein diacetate (DCFDA, $50 \mu \mathrm{M})$ for $15 \mathrm{~min}$ and then stimulated with or without Mtb-Ag $(10 \mu \mathrm{g} / \mathrm{ml}) \pm$ PGE2 $(2 \mu \mathrm{M})$ for 60 min. Finally, DCFDA fluorescence was evaluated to monitor ROS production by flow cytometry. Left panel: Bars represent the mean values of the mean fluorescence intensity relative to control (rMFI) of neutrophils \pm SEM. Right panel: a representative histogram is shown (Control: non-stained cells). 
a.

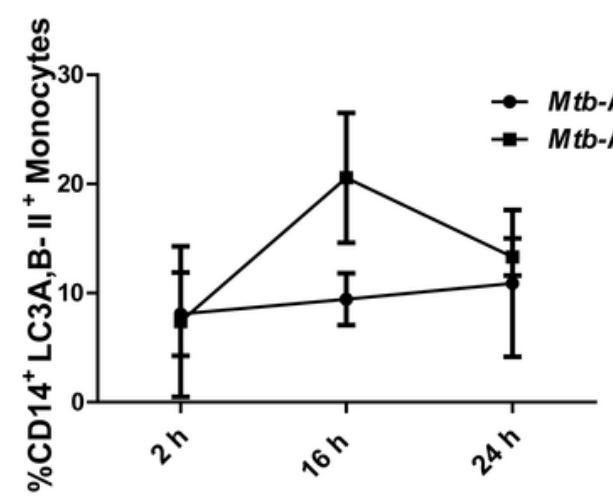

c.

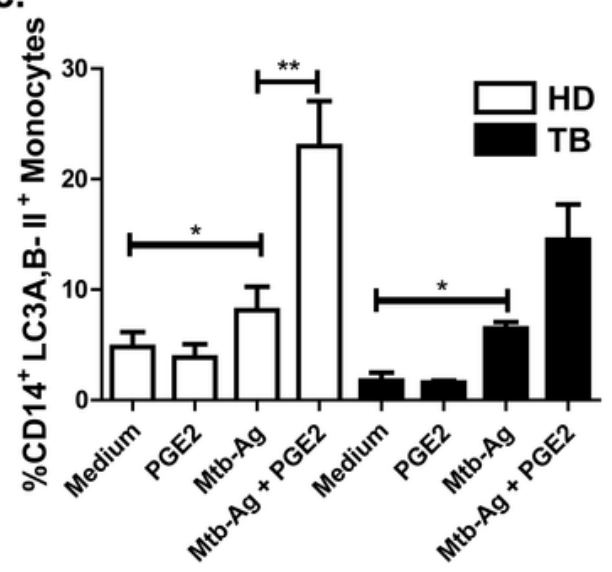

e.

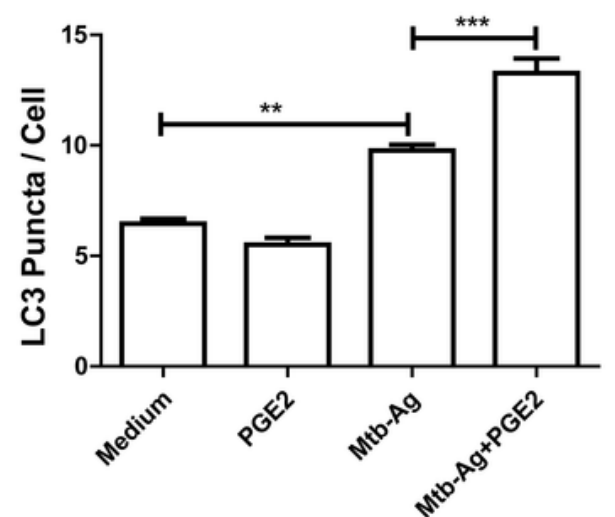

f.

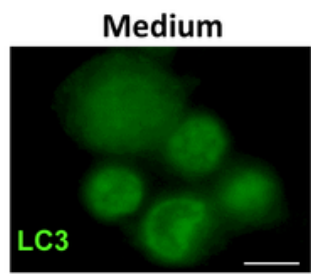

b.

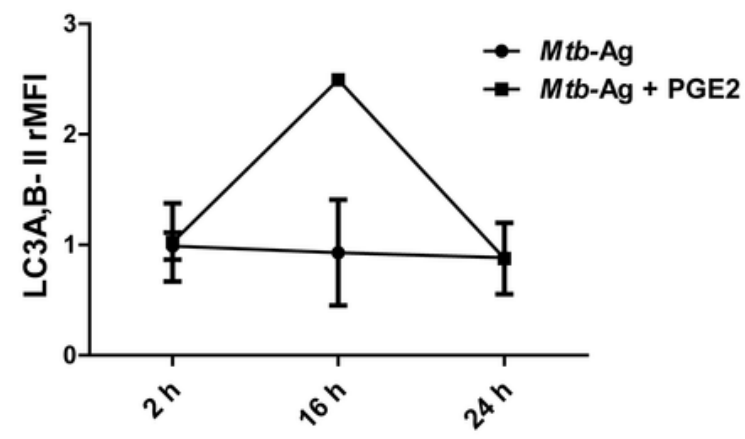

d.

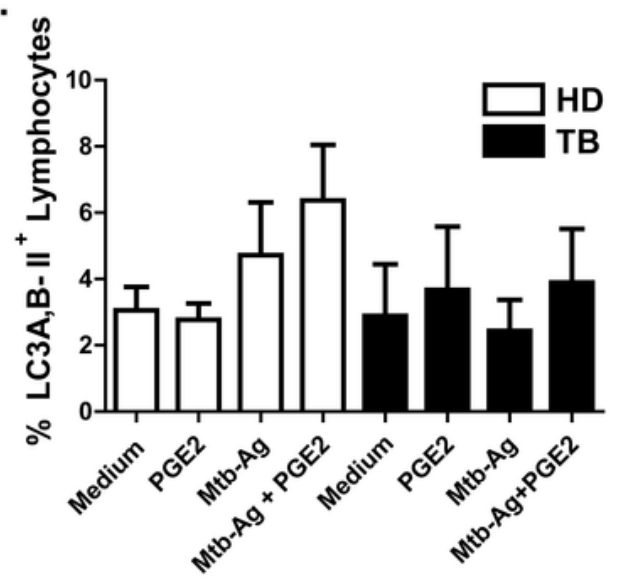

g.

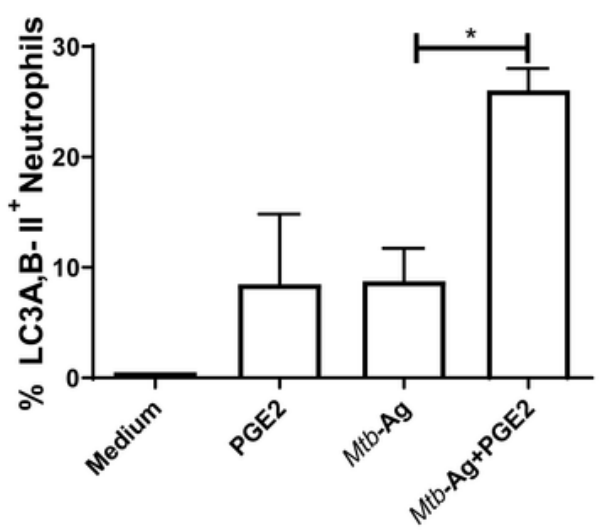

Mtb-Ag

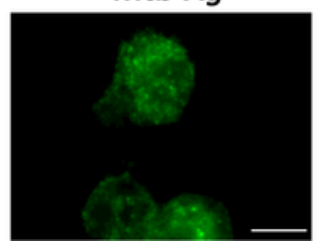

$M t b-A g+$ PGE2

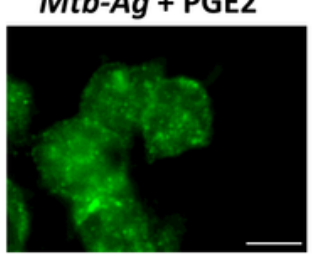

Figure 6

PGE2 promotes autophagy in Mtb-Ag-stimulated cells from HD and TB patients. Adherent cells from HD $(n=5)$ and TB patients $(T B, n=4)$ were stimulated with $M t b-A g(10 \mu g / m l)$ in the presence or absence of PGE2 $(2 \mu \mathrm{M})$ at the indicated time points. The levels of autophagy were then evaluated by intracellular flow cytometry. (A) Percentage of CD14+LC3A, B-Il+ cells \pm SEM; (B) Mean fluorescence intensity (MFI) of CD14+LC3A, B-II+ cells \pm SEM. (C, D) Bars represent the percentage of (C) monocytes CD14+ LC3A, B-II+ 
and (D) lymphocytes CD3+ LC3A, B-II+ after 16 hours of stimulation. (E) PBMC from HD $(n=4)$ were incubated at $2 \times 106$ cells $/ \mathrm{ml}$ for 16 hours to allow adherence of monocytes. Afterwards, the cells were stimulated with Mtb-Ag $(10 \mu \mathrm{g} / \mathrm{ml})$ in the presence or absence of PGE2 $(2 \mu \mathrm{M})$ for 16 hours. Autophagy levels were then evaluated by immunofluorescence against LC3 on monocytes. Bars represent the mean values of LC3 puncta per cell \pm SEM. (F) A representative experiment is shown. (G) Human purified neutrophils from HD $(n=7)$ were stimulated with Mtb-Ag $(10 \mu \mathrm{g} / \mathrm{ml})$ in the presence or absence of PGE2 (2 $\mu \mathrm{M})$ for 2 hours. The levels of autophagy were then evaluated by intracellular flow cytometry. Statistical differences were calculated using one-way ANOVA and post hoc Dunnett's multiple comparison test. * $p<0.05, * \star p<0.01, * \star \star p<0.001$.

a.

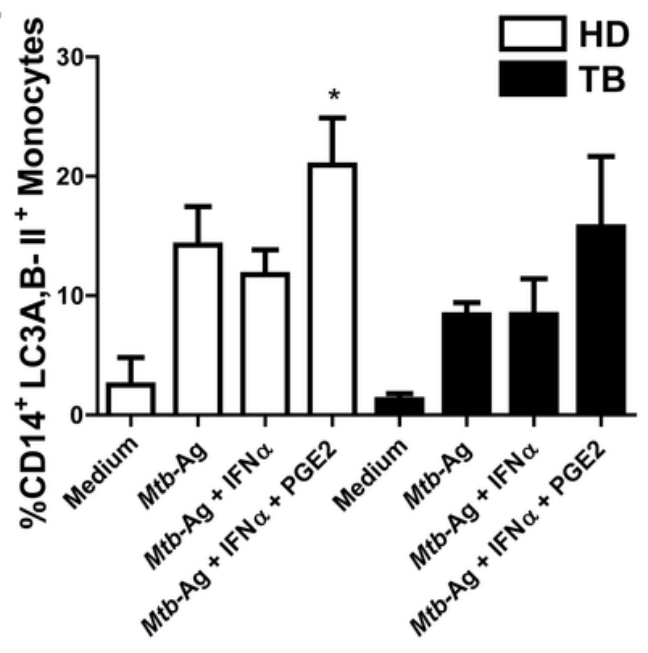

b.

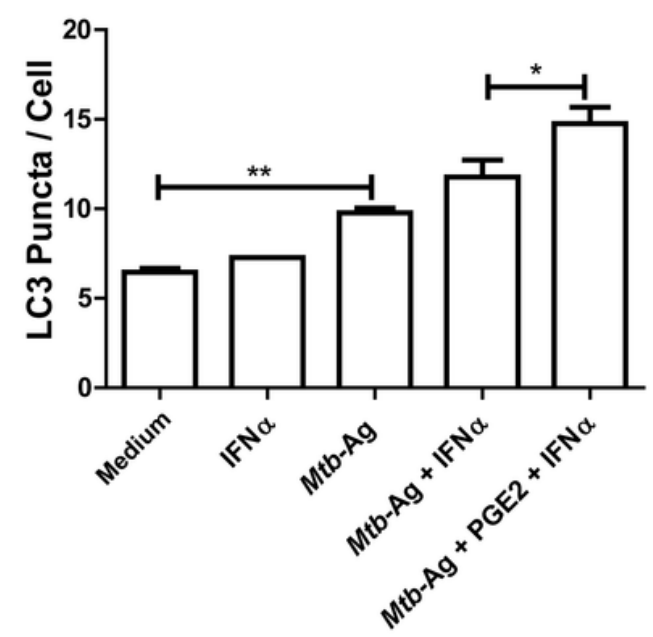

c. Medium
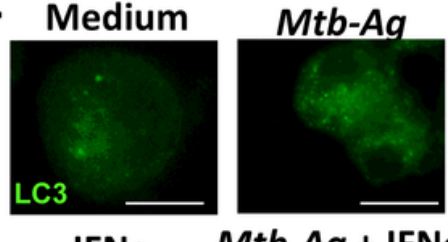

$M t b-A g+$ IFN $\alpha$
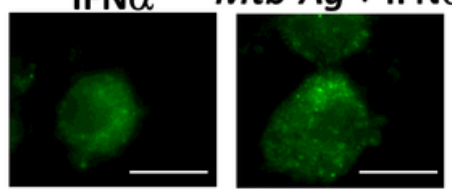

$M t b-A g+$ IFN $\alpha+$ PGE2

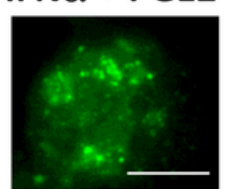

Figure 7

Effect of IFNa on the regulation of autophagy in human monocytes. PBMC from HD $(n=4)$ and TB patients $(n=5)$ were incubated at $2 \times 106$ cells/ml during $16 \mathrm{~h}$ to allow adherence of monocytes. Afterwards, the cells were stimulated with Mtb-Ag $(10 \mu \mathrm{g} / \mathrm{ml})$ in the presence or absence of IFNa (10 $\mathrm{ng} / \mathrm{ml})$ with or without PGE2 $(2 \mu \mathrm{M})$ during $16 \mathrm{~h}$. Autophagy levels were evaluated by (A) flow cytometry against intracellular saponin-resistant LC3A, B-II in CD14+ cells and by (B) immunofluorescence against LC3B in monocytes. (C) Representative images of one experiment are shown. Bars represent the mean values of LC3 puncta per cell \pm SEM. * $p<0.05$, $* \star p<0.01$. Statistical differences were calculated using one-way ANOVA and post hoc Dunnett multiple comparison test.

\section{Supplementary Files}

This is a list of supplementary files associated with this preprint. Click to download.

- SupplementaryMaterialPellegrinietal.pdf 